\title{
Estudando o Impacto das Atitudes de Agentes Pedagógicos em Ambientes de Aprendizagem: um Mapeamento Sistemático
}

\author{
Otávio B. Azevedo ${ }^{1}$, Patrícia A. Jaques ${ }^{1}$ \\ ${ }^{1}$ Programa de Pós-Graduação em Computação Aplicada (PPGCA) \\ Universidade do Vale do Rio dos Sinos (UNISINOS), São Leopoldo - RS - Brazil \\ obazevedo@edu.unisinos.br, pjaques@unisinos.br
}

\begin{abstract}
Teachers' affective attitudes can have a positive or negative impact on student learning. Therefore, it's a good practice to consider them in the design of animated pedagogic agents. However, there are no studies that present a global view about the influence of agents' attitudes (i.e., rude, empathic, etc) on students. A systematic mapping was conducted with the goal of providing an overview of the main attitudes of affective pedagogical agents and their effects on students. A total of 62 articles were collected from the main international databases, of which 15 were selected for the complete analysis. As a result, scientific articles from the current state-of-the-art of Animated Pedagogical Agents that establish relationships of empathy, politeness, rudeness, and rapport with their students was presented. Most studies focus on maximizing learning and engagement from empathic agents.
\end{abstract}

Resumo. As atitudes afetivas dos professores podem ter um impacto positivo ou negativo na aprendizagem dos alunos. Assim sendo, é uma boa prática considerá-las na concepção de agentes pedagógicos animados. Entretanto, ainda não existem estudos que apresentam uma visão global sobre a influência das atitudes (por exemplo, rude, empática, etc) dos agentes nos alunos. Dessa maneira, um mapeamento sistemático foi conduzido com o objetivo de fornecer uma visão geral de quais são as principais atitudes dos agentes pedagógicos afetivos e os seus efeitos nos estudantes. Foram coletados 62 artigos das principais bases de dados internacionais, dois quais 15 foram selecionados para a análise completa. Como resultado, foram apresentados os artigos científicos do atual estado da arte dos Agentes Pedagógicos Animados que estabelecem relações de empatia, polidez, rudez e rapport com os seus alunos. A maioria dos estudos concentra-se em maximizar a aprendizagem e o engajamento a partir de agentes empáticos que criam relações afetivas com os alunos.

\section{Introdução}

Uma boa comunicação entre o professor e o aluno é fundamental para que ocorra aprendizagem, seja presencial ou à distância [Morreale and Pearson 2008]. Esse princípio também é válido para os Sistemas Tutores Inteligentes e Ambientes Virtuais de Aprendizagem, que geralmente se comunicam com o estudante através de texto e/ou áudio. No entanto, os alunos podem sentir falta da presença do professor, de maneira que a comunicação não seja tão eficaz como em uma aula presencial. Os Agentes Pedagógicos Animados (APAs) podem ajudar a minimizar este problema, trazendo uma representação 
VIII Congresso Brasileiro de Informática na Educação (CBIE 2019)

Anais do XXX Simpósio Brasileiro de Informática na Educação (SBIE 2019)

virtual do professor que pode se comunicar com o estudante pela fala, gestos e expressões faciais antropomórficas [Jaques and Vicari 2005].

Estudos mostraram que os APAs se tornam mais eficientes quando estabelecem uma relação afetiva com os seus alunos como se fossem um tutor humano, fazendo com que eles aprendam mais [Jaques et al. 2008, Wang et al. 2010, Karacora et al. 2012]. Esse é um tema de interesse da área de pesquisa de Computação Afetiva, que estuda e desenvolve sistemas computacionais que podem reconhecer, interpretar, processar e simular afetos humanos [Picard 2000]. Ainda segundo a autora, a máquina consegue interpretar o estado emocional dos seres humanos e adaptar o seu comportamento a eles, dando uma resposta mais apropriada a essas emoções.

As primeiras pesquisas sobre agentes pedagógicos afetivos começaram há algumas décadas [Lester et al. 1998, Cooper et al. 1999], porém o interesse por essa área vem aumentando consideravelmente [Mohanty 2016, Dinçer and Doğanay 2017, Terzidou et al. 2018], inclusive em contextos não educacionais, como é o caso das assistentes pessoais Cortana, Google Assistant e Siri [Hoy 2018]. Entretanto, ainda não existem estudos que apresentam uma visão geral sobre o impacto das atitudes dos agentes afetivos no aprendizado, engajamento e estados afetivos dos alunos. Com o propósito de preencher essa lacuna, foi realizado um Mapeamento Sistemático dos últimos 12 anos de trabalhos que envolvem os APAs e a Computação Afetiva. O Mapeamento Sistemático consiste em identificar, avaliar e interpretar todos os trabalhos relevantes para uma determinada área de pesquisa [Petersen et al. 2008].

Segundo [Scherer 2005], a postura afetiva adotada por um indivíduo em relação ao outro em uma determinada situação é denominada postura interpessoal. Entretanto, no contexto dos Agentes Pedagógicos Animados, essa é a definição de atitude [Ferguson 1992]. Como este trabalho está na área dos APAs, o termo atitude foi utilizado ao invés de postura interpessoal. Assim sendo, o objetivo deste estudo é analisar a influência das atitudes de agentes pedagógicos animados nos estudantes, que contam com uma ou mais dessas atitudes: empatia, polidez, rudez e rapport. A empatia pode ser definida como a capacidade de compreender o outro; uma pessoa empática é capaz de se colocar no lugar do outro e compartilhar suas emoções [Eisenberg and Strayer 1990]. Assim sendo, o rapport é o resultado da combinação de empatia com a polidez (gentileza) ou rudez (grosseria) [Spencer-Oatey 2005]. Ele promove uma sensação de sincronia entre duas ou mais pessoas, proporcionando um relacionamento mais agradável [Gratch et al. 2006].

Os próximos conteúdos deste artigo estão organizados da seguinte forma: a Seção 2 descreve como o mapeamento foi realizado e quais são as nossas questões de pesquisa. $\mathrm{Na}$ Seção 3 foram apresentados os resultados da análise dos artigos, com as respostas de cada uma das questões anteriores (a discussão desses resultados está disponível na Seção 4). A Seção 5 discute as possíveis ameaças à validade deste trabalho. Por último, a Seção 6 apresenta as conclusões deste trabalho.

\section{O Processo do Mapeamento Sistemático}

O mapeamento conduzido foi baseado no processo descrito por [Petersen et al. 2008], que definiram cinco etapas essenciais a serem seguidas: (1) definição de questões de pesquisa, (2) realização da pesquisa e estudos primários relevantes, (3) triagem dos documentos, (4) keywording dos resumos, e (5) extração de dados e mapeamento. 
VIII Congresso Brasileiro de Informática na Educação (CBIE 2019)

Anais do XXX Simpósio Brasileiro de Informática na Educação (SBIE 2019)

Considerando que as questões de pesquisa devem exemplificar os objetivos do estudo de mapeamento, as seguintes questões foram elaboradas:

$\mathbf{Q P}_{1}$ : Em quais contextos e níveis educacionais os APAs com emoções são utilizados?

$\mathbf{Q P}_{2}$ : Quais são as relações afetivas que os APAs estabelecem com os alunos?

$\mathbf{Q P}_{3}$ : Quais são os tipos de ambientes de aprendizagem empregados?

QP 4 : Quais são os métodos e tipos de dados empregados para a avaliação dos trabalhos?

QP 5 : Quais são os perfis dos participantes nas avaliações?

QP $\mathbf{P}_{6}$ : Quais são as variáveis dependentes consideradas nas avaliações? E quais foram os resultados obtidos?

$\mathbf{Q P}_{7}$ : Os estudos buscaram detectar ou regular os estados afetivos dos participantes? Se sim, qual foi o impacto dessas ações?

O processo de seleção dos artigos foi dividido em quatro etapas: (1) busca de artigos a partir de um conjunto de palavras-chave, (2) seleção de resumos, (3) seleção de textos completos e (4) extração de informações-chave para classificar os trabalhos

Uma busca de artigos publicados em inglês a partir de 2008 foi realizada nas bases da ACM e IEEE (não foram encontrados artigos em português que estivessem de acordo com os critérios deste trabalho, mesmo traduzindo as palavras-chave utilizadas na pesquisa), e também foi utilizada a ferramenta do Google Acadêmico. As pesquisas foram efetuadas a partir de combinações de três termos-chave, conforme mostra a Tabela 1. Os termos que estão em negrito foram combinados com todas as palavras da mesma linha, totalizando oito combinações (células A1 até B4).

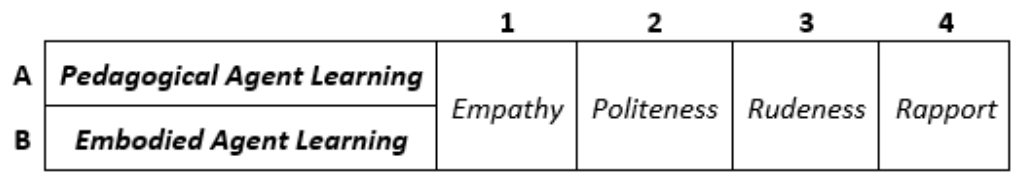

Tabela 1. Combinações de palavras-chave para as pesquisas de artigos

A segunda etapa contou com a leitura dos resumos dos 62 artigos encontrados. A análise foi baseada em três critérios: (1) relação com a área da aprendizagem, (2) utilização de agentes pedagógicos animados e (3) relação afetiva do agente com o aluno a partir de empatia, polidez/rudez e/ou rapport (os trabalhos que não envolveram pelo menos um desses quatro estados afetivos foram desconsiderados). Essa avaliação teve como resultado um conjunto de 29 artigos a serem analisados na terceira etapa (47\% do total).

O terceiro estágio contou com a leitura completa dos artigos selecionados na etapa anterior (a lista completa de artigos pode ser visualizada em: https: / t inyurl. com/rev-artigos). Nessa análise foram desconsiderados os artigos short-paper devido à falta de informações mais completas, e os trabalhos que não fizeram uma avaliação também foram desclassificados. Dessa maneira, 15 artigos foram selecionados para a etapa final (24\% do total e $52 \%$ dos que tiveram os resumo aceito).

A Figura 1 ilustra os artigos selecionados e não selecionados por ano. Observa-se que a maioria dos trabalhos (67\%) foram publicados entre 2008 e 2012. 
VIII Congresso Brasileiro de Informática na Educação (CBIE 2019)

Anais do XXX Simpósio Brasileiro de Informática na Educação (SBIE 2019)

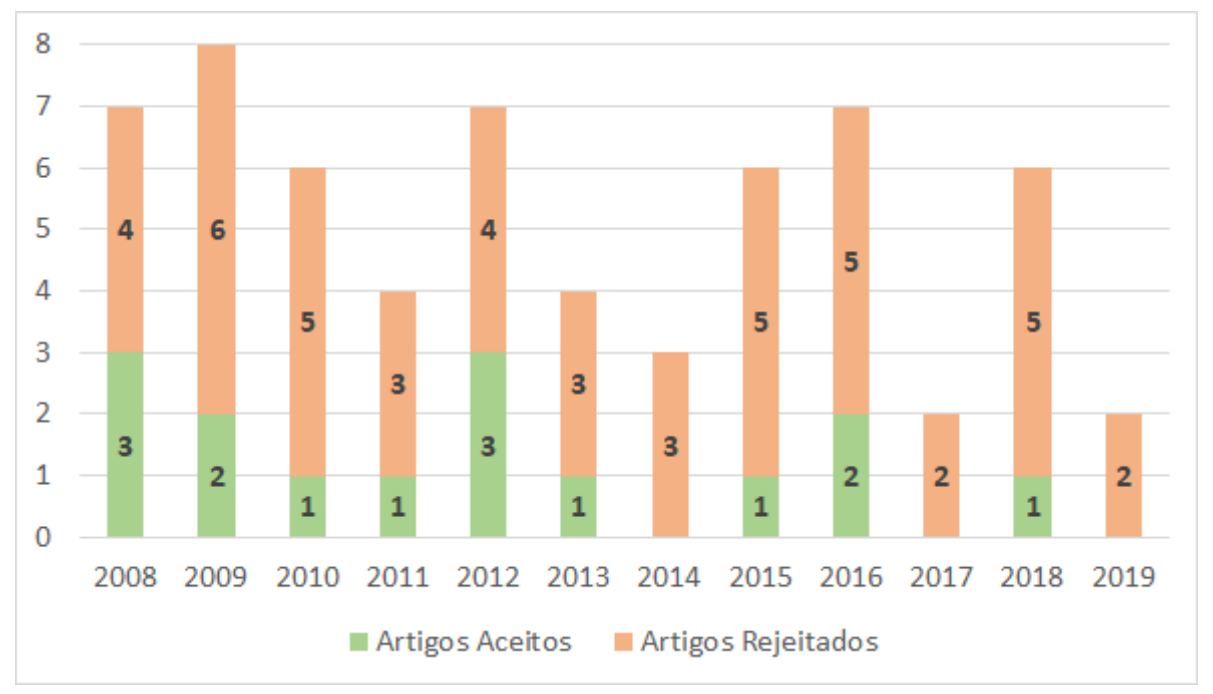

Figura 1. Seleção de artigos por ano

Na quarta e última etapa os 15 artigos selecionados foram lidos novamente, a fim de extrair as informações necessárias para responder às sete questões de pesquisa definidas anteriormente.

\section{Resultados}

Nesta seção estão descritos os resultados obtidos a partir da leitura e análise dos 15 trabalhos selecionados, a fim de responder às questões de pesquisa.

$\left(\mathbf{Q P}_{1}\right)$ Contextos e Níveis Educacionais: Observa-se na Figura 2 que a maioria dos trabalhos (33\%) são voltados para o ensino superior, e apenas um deles (7\%) é direcionado para o aprendizado de idiomas. Em segundo lugar, quatro artigos (27\%) estão relacionados com a educação em geral, que não especificam o nível educacional mais apropriado. Esses estudos limitam-se a discutir os efeitos positivos e negativos de agentes pedagógicos afetivos na educação. Os demais estudos envolveram conteúdos do ensino fundamental (20\%) e médio (13\%). Nenhum dos trabalhos foi voltado para a pré-escola ou para estudantes com necessidades especiais.

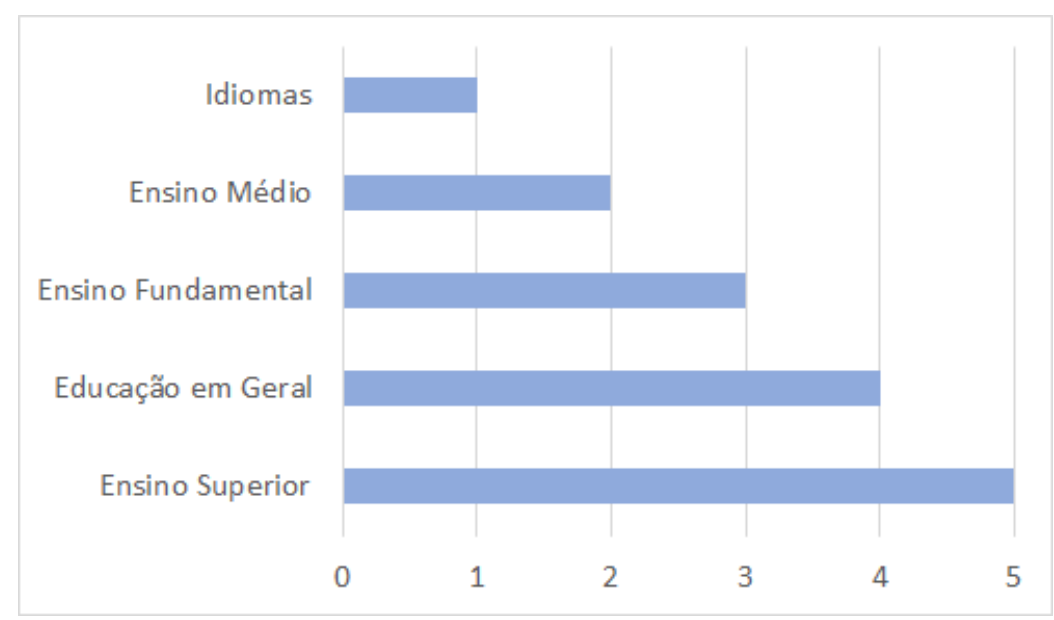

Figura 2. Trabalhos classificados de acordo com o contexto e nível educacional 
VIII Congresso Brasileiro de Informática na Educação (CBIE 2019)

Anais do XXX Simpósio Brasileiro de Informática na Educação (SBIE 2019)

$\left(\mathbf{Q P}_{2}\right)$ Relações Afetivas dos Agentes com os Usuários: A maior parte dos estudos (12 trabalhos, representando um total de 80\%) utilizaram agentes empáticos e com comportamento polido/rude, conforme mostra a Figura 3. Os outros três trabalhos (20\%) contaram com agentes animados que expressam o estado de rapport.

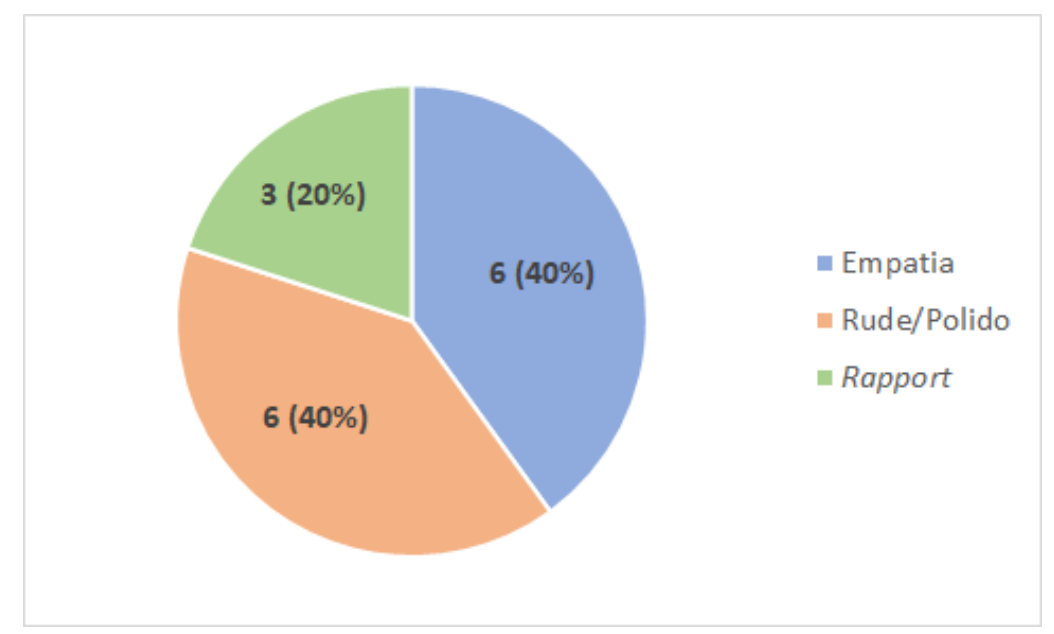

Figura 3. Relações afetivas dos agentes pedagógicos animados com os alunos

$\left(\mathbf{Q P}_{3}\right)$ Tipos de Ambientes de Aprendizagem: Observa-se na Figura 4 que os ambientes mais utilizados pelos pesquisadores foram os Sistemas Tutores Inteligentes e os Ambientes Virtuais de Aprendizagem, correspondendo à $80 \%$ dos trabalhos. Isso se deve ao fato de que os pesquisadores que trabalham com agentes pedagógicos animados são da área da inteligência artificial e tutores inteligentes, uma vez que eles precisam desses recursos para tornar os APAs mais eficientes e eficazes [Maes 1995]. Além disso, um dos trabalhos não utilizou um sistema computacional para o experimento, e os outros dois utilizaram um Jogo Sério e um Sistema de Diálogo Falado, respectivamente.

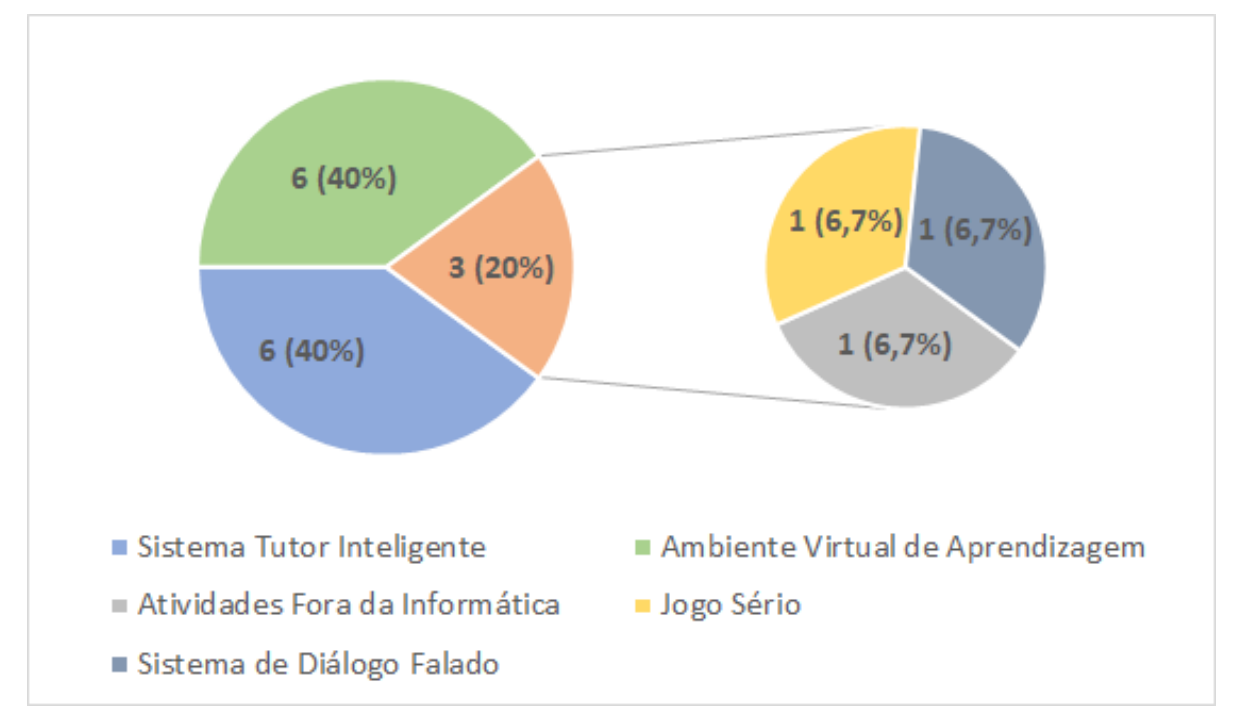

Figura 4. Tipos de ambientes de aprendizagem utilizados pelos trabalhos

$\left(\mathbf{Q P}_{4}\right)$ Métodos de Pesquisa e Tipos de Dados: Em relação ao método de pesquisa, todos os 15 trabalhos selecionados realizaram uma avaliação empírica a partir de um experimento quantitativo. Quanto aos tipos de dados, a maioria dos pesquisadores $(60 \%)$ 
VIII Congresso Brasileiro de Informática na Educação (CBIE 2019)

Anais do XXX Simpósio Brasileiro de Informática na Educação (SBIE 2019)

utilizaram testes de conhecimento para coletar os dados. Os questionários foram o segundo recurso mais utilizado (53\% dos casos, considere que um trabalho pode utilizar mais de uma fonte de dados), seguidos das entrevistas, que foram utilizadas por dois trabalhos (13\% do total).

(QP $)$ Perfis dos Participantes (idades e gêneros): Observa-se na Figura 5 que a maioria dos participantes $(50 \%)$ está dividida entre duas categorias: um quarto está na faixa etária de 10 a 15 anos e os outros $25 \%$ não tiveram a idade especificada. Em segundo lugar também houve um empate entre as faixas etária de 15 a 20 anos e 20 a 30 anos, cada uma corresponde $19 \%$ do total. Os $12 \%$ restantes correspondem aos alunos com 30 a 50 anos de idade. Além disso, há uma quantidade similar de estudantes do gênero feminino e masculino ( $45 \%$ e $42 \%$ respectivamente), e $13 \%$ não tiveram o gênero confirmado pelos autores.

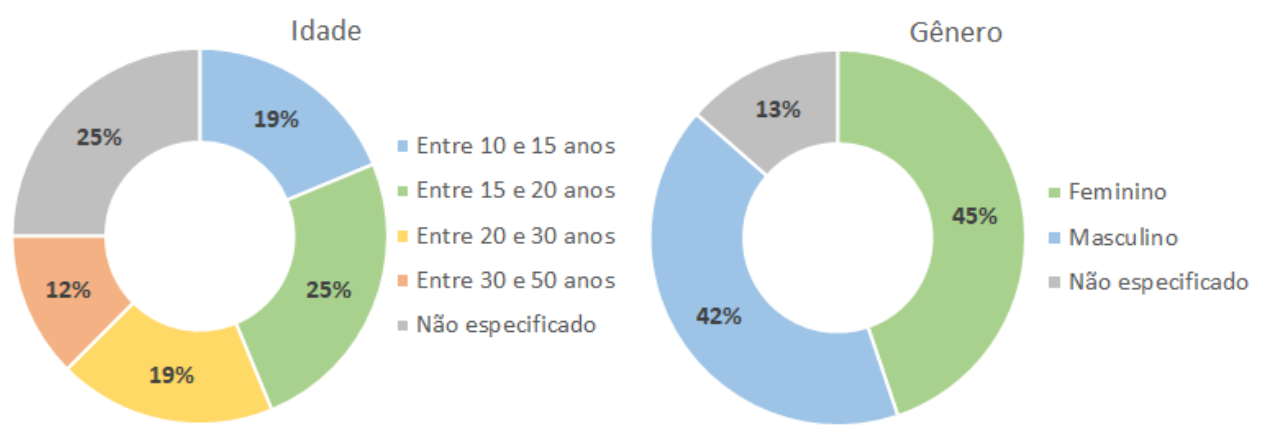

Figura 5. Perfis dos participantes nas avaliações

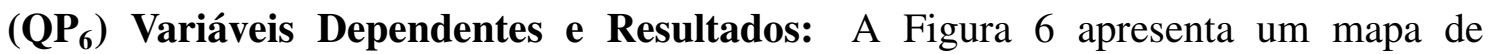
distribuição dos trabalhos, com base em suas variáveis dependentes (eixo y) e as relações afetivas que os APAs estabelecem com os alunos (eixo x). Observa-se que a maioria dos estudos (11 dos 15 trabalhos) teve como objetivo melhorar o aprendizado dos estudantes e, desses 11 trabalhos, quatro também tiveram o objetivo de engajar os alunos. Além disso, dois trabalhos visaram detectar e regular os estados afetivos dos alunos. Também é possível constatar que os agentes empáticos e polidos/rudes foram os mais utilizados para promover a aprendizagem. Finalmente, os agentes empáticos e com rapport foram os mais utilizados para aumentar o engajamento.

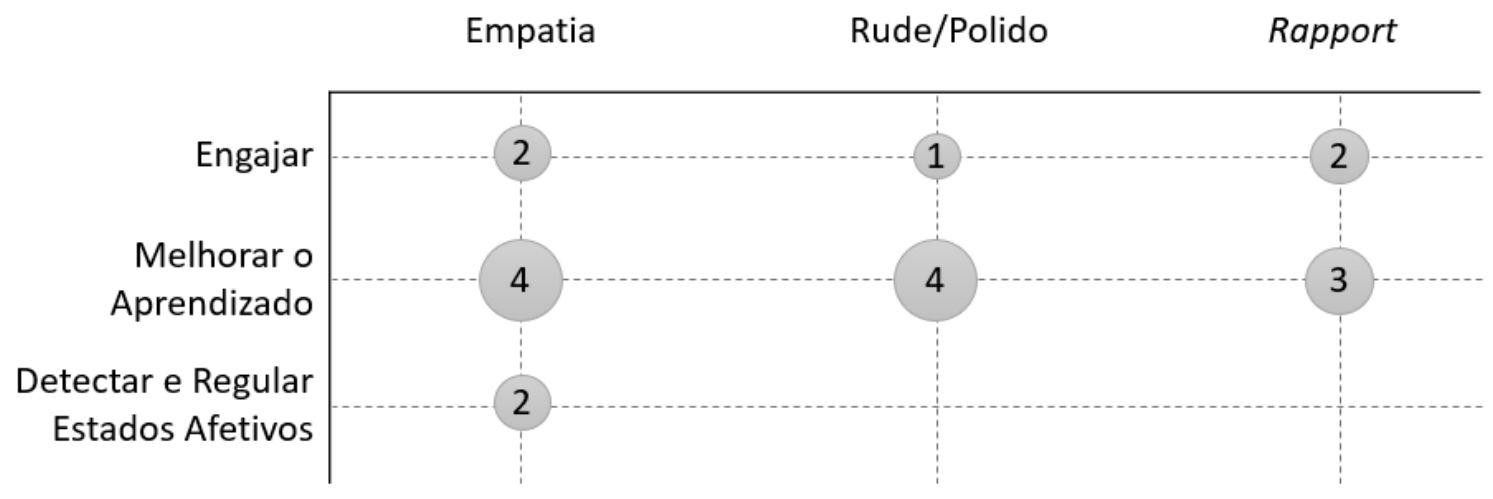

Figura 6. Mapa de distribuição dos trabalhos 
Em relação aos resultados ${ }^{1}$, todos os estudos que envolveram agentes empáticos tiveram êxito em seus objetivos, o que indica que adicionar empatia nos APAs é uma das melhores estratégias para engajar os alunos e promover a aprendizagem. Além disso, os agentes polidos foram mais aceitos e ajudaram mais os alunos do que os agentes rudes. Porém, no caso do trabalho que envolveu atividades de química, o APA educado foi mais eficiente somente para os alunos com menor conhecimento prévio. Finalmente, os agentes com rapport ajudaram a melhorar o desempenho e engajamento dos alunos na maior parte dos trabalhos.

$\left(\mathbf{Q P}_{7}\right)$ Detecção ou Regulação dos Estados Afetivos dos Usuários: Foi realizado um levantamento dos trabalhos que detectaram ou regularam os estados afetivos dos alunos, a fim de verificar os impactos dessas ações na aprendizagem e engajamento. Observa-se na Figura 6 da seção anterior que somente dois trabalhos realizaram essa detecção/regulação de estados afetivos.

O primeiro trabalho buscou detectar as emoções de felicidade, tristeza e medo dos alunos. Quando eles estavam felizes, o agente exibia um comportamento de empatia e felicidade; porém, se os alunos estivessem tristes ou com medo, o APA demonstrava um comportamento empaticamente encorajador. Os resultados indicaram que o agente conseguiu manter a emoção dos estudantes quando estavam felizes, e também foi capaz de regular um estado emocional de medo ou tristeza para um estado neutro.

Os autores do outro trabalho tiveram problemas para conhecer os estados afetivos dos alunos, uma vez que eles não expressaram suas emoções conforme o solicitado. Uma possível razão para esse problema foi o curto período de tempo da sessão.

\section{Discussão dos Resultados}

Os trabalhos analisados envolveram quase todos os níveis educacionais, desde o ensino fundamental até o ensino superior (ver figura 2). Porém, estão em falta os sistemas direcionados para a pré-escola. Além disso, não foram encontrados ambientes de aprendizagem com agentes pedagógicos afetivos que são acessíveis para portadores de necessidades especiais. No contexto da afetividade, esses dois públicos podem reagir de maneira diferente no contato com agentes afetivos em relação aos demais estudantes [Babad et al. 1987, Garay et al. 2006]. Dessa maneira, seria interessante se houvesse estudos que confirmassem ou refutassem essa hipótese. Em relação à educação em geral, é preciso lembrar que a pré-escola é a base para todos os outros níveis de ensino, e quase um quarto da população brasileira possui necessidades especiais ${ }^{2}$, o que ressalta a importância de trabalhos que envolvam esses públicos específicos.

A Figura 3 nos mostrou que quase a metade dos trabalhos utilizaram agentes empáticos, e todos eles tiveram sucesso em seus objetivos (ver Seção 3). Esses resultados são compatíveis com o trabalho de [Albuquerque 2010], que fez uma entrevista com 40 alunos do ensino médio e fundamental. Os resultados indicaram que o relacionamento afetivo do professor com o aluno é um dos fatores mais importante na sala de aula, e a principal característica de um bom professor é a empatia. Porém, esses trabalhos

\footnotetext{
${ }^{1} \mathrm{~A}$ tabela completa dos resultados encontrados pelos estudos pode ser visualizada em: https:// tinyurl.com/tab-resultados.

${ }^{2}$ Segundo o IBGE, 45 milhões de brasileiros possuem algum tipo de deficiência, o que corresponde à quase $24 \%$ da população. Esses dados estão disponíveis em: https : / / i nyurl . com/ibge-dados.
} 
VIII Congresso Brasileiro de Informática na Educação (CBIE 2019)

Anais do XXX Simpósio Brasileiro de Informática na Educação (SBIE 2019)

não consideraram as características dos alunos (como a personalidade), portanto não é possível afirmar que as atitudes empáticas são as melhores para todos os alunos. Além disso, os resultados de uma boa parte dos experimentos indicaram que o impacto positivo das atitudes dos agentes na aprendizagem dos alunos era maior quando eles tinham pouco conhecimento prévio nos conteúdos estudados.

Por último, foi encontrada uma contradição entre os resultados dos trabalhos de [Krämer et al. 2016] e [Wang and Gratch 2009], que envolveram agentes com rapport. O primeiro trabalho identificou um aumento no esforço e desempenho dos alunos que contaram com o APA com rapport, porém não houve experiência subjetiva (motivação e relacionamento). Entretanto, o outro trabalho teve o resultado contrário: o agente com rapport proporcionou uma experiência subjetiva, mas não ajudou os alunos a terem um melhor desempenho. Os autores desse segundo trabalho sugeriram que APAs com rapport podem não ter um impacto significativo na aprendizagem de conceitos fáceis, mas sim nos conceitos difíceis. Dessa maneira, seria interessante reaplicar esses experimentos com conteúdos fáceis e difíceis para validar a hipótese de [Wang and Gratch 2009] e verificar se essa contradição aconteceria novamente.

\section{Ameaças à Validade}

As questões de pesquisa e os critérios de inclusão e exclusão de trabalhos foram definidos antes de iniciar o mapeamento, garantindo assim um processo de seleção imparcial. Além disso, foi utilizado um conjunto limitado de bases de dados (ver seção 2). Dessa maneira, é possível que estudos relevantes não tenham sido considerados. Por fim, a coerência do sistema de classificação elaborado pode significar uma ameaça à validade, pois o conhecimento necessário para elaborá-lo normalmente é obtido somente ao final da seleção [Pretorius and Budgen 2008].

\section{Conclusão}

O objetivo deste trabalho foi realizar um mapeamento sistemático sobre os estudos que envolvem Agentes Pedagógicos Afetivos na Educação, a partir de pesquisas nas principais bases de dados internacionais (ACM e IEEE) e na plataforma do Google Acadêmico. Foi reunido um conjunto de 62 trabalhos, dos quais 15 foram selecionados a partir dos nossos critérios de inclusão e exclusão definidos na Seção 2. Com base nesses trabalhos, foi possível responder as nossas questões de pesquisa (também definidas na Seção 2).

Os resultados desse mapeamento indicaram que a maior parte dos trabalhos (um terço do total) foram voltados para estudantes universitários, conforme mostra a Figura $2\left(\mathbf{Q P}_{\mathbf{1}}\right)$. Além disso, $80 \%$ dos trabalhos utilizaram agentes empáticos e com comportamento rude/polido ( $\mathbf{Q} \mathbf{P}_{\mathbf{2}}$ - ver Figura 3). Em relação aos ambientes de aprendizagem, os mais utilizados foram os Sistemas Tutores Inteligentes e Ambientes Virtuais de Aprendizagem, conforme mostra a Figura $4\left(\mathbf{Q P}_{\mathbf{3}}\right)$. Respondendo a $\mathbf{Q P} \mathbf{P}_{\mathbf{4}}$, todos os autores realizaram um experimento, e o principal artefato para coletar dados foi o teste de conhecimento (utilizado por $60 \%$ dos trabalhos). Quanto ao perfil dos participantes, a maioria era adolescente (com idade de 10 a 15 anos) e havia uma quantidade similar de estudantes de gênero feminino e masculino ( $\mathbf{Q P}_{5}$ - ver Figura 5)). Além disso, o principal objetivo dos trabalhos foi melhorar o aprendizado dos alunos (ver Figura 6), sendo que os agentes empáticos e polidos tiveram os melhores resultados $\left(\mathbf{Q P}_{\mathbf{6}}\right)$. Por último, houve um trabalho 
VIII Congresso Brasileiro de Informática na Educação (CBIE 2019)

Anais do XXX Simpósio Brasileiro de Informática na Educação (SBIE 2019)

que realizou a detecção de três estados afetivos dos alunos (felicidade, tristeza e medo), que teve como objetivo manter a emoção do aluno se ele estivesse feliz, ou regular para uma emoção neutra caso ele estivesse triste ou com medo $\left(\mathbf{Q P}_{\mathbf{7}}\right)$.

Poucos estudos trabalharam com a regulação de estados afetivos dos alunos, e existem poucos sistemas acessíveis para portadores de necessidades especiais. Além disso, não foram encontrados trabalhos que consideram o conhecimento prévio do aluno e suas características afetivas, como a personalidade. Assim, essa seria uma oportunidade interessante para desenvolver mais trabalhos para esses contextos. Uma outra contribuição dessa pesquisa é a disponibilização do primeiro mapeamento sistemático na área de agentes pedagógicos que considera o impacto de suas atitudes nos estudantes.

\section{Agradecimentos}

O presente trabalho foi realizado com apoio da Capes (bolsa de mestrado PROSUC, processo STICAMSUD 18-STIC-03), FAPERGS (Processo 17/2551-0001203-8) e CNPq (processo 309218/2017-9).

\section{Referências}

Albuquerque, C. (2010). Processo ensino-aprendizagem: características o professor eficaz. Millenium, pages 55-71.

Babad, E., Bernieri, F., and Rosenthal, R. (1987). Nonverbal and verbal behavior of preschool, remedial, and elementary school teachers. American Educational Research Journal, 24(3):405-415.

Cooper, B., Brna, P., and Martins, A. (1999). Effective affective in intelligent systemsbuilding on evidence of empathy in teaching and learning. In International Workshop on Affective Interactions, pages 21-34. Springer.

Dinçer, S. and Doğanay, A. (2017). The effects of multiple-pedagogical agents on learners' academic success, motivation, and cognitive load. Computers \& Education, 111:74-100.

Eisenberg, N. and Strayer, J. (1990). Empathy and its development. CUP Archive.

Ferguson, I. A. (1992). Touring machines: Autonomous agents with attitudes. Computer, 25(5):51-55.

Garay, N., Cearreta, I., López, J. M., and Fajardo, I. (2006). Assistive technology and affective mediation. Human Technology: an Interdisciplinary Journal on Humans in ICT Environments.

Gratch, J., Okhmatovskaia, A., Lamothe, F., Marsella, S., Morales, M., van der Werf, R. J., and Morency, L.-P. (2006). Virtual rapport. In International Workshop on Intelligent Virtual Agents, pages 14-27. Springer.

Hoy, M. B. (2018). Alexa, siri, cortana, and more: An introduction to voice assistants. Medical reference services quarterly, 37(1):81-88.

Jaques, P. A., Lehmann, M., and Jaques, K. S. F. (2008). Avaliando a efetividade de um agente pedagógico animado emocional. In Brazilian Symposium on Computers in Education (Simpósio Brasileiro de Informática na Educação-SBIE), volume 1, pages $145-154$. 
VIII Congresso Brasileiro de Informática na Educação (CBIE 2019)

Anais do XXX Simpósio Brasileiro de Informática na Educação (SBIE 2019)

Jaques, P. A. and Vicari, R. M. (2005). Estado da arte em ambientes inteligentes de aprendizagem que consideram a afetividade do aluno. Revista informática na educação: teoria \& prática, 8(1):15-38.

Karacora, B., Dehghani, M., Kramer-Mertens, N., and Gratch, J. (2012). The influence of virtual agents' gender and rapport on enhancing math performance. In Proceedings of the Annual Meeting of the Cognitive Science Society, volume 34.

Krämer, N. C., Karacora, B., Lucas, G., Dehghani, M., Rüther, G., and Gratch, J. (2016). Closing the gender gap in stem with friendly male instructors? on the effects of rapport behavior and gender of a virtual agent in an instructional interaction. Computers \& Education, 99:1-13.

Lester, J. C., Towns, S. G., and Fitzgerald, P. J. (1998). Achieving affective impact: Visual emotive communication in lifelike pedagogical agents. International Journal of Artificial Intelligence in Education (IJAIED), 10:278-291.

Maes, P. (1995). Agents that reduce work and information overload. In Readings in human-computer interaction, pages 811-821. Elsevier.

Mohanty, A. (2016). Affective pedagogical agent in e-learning environment: A reflective analysis. Creative Education, 7(04):586.

Morreale, S. P. and Pearson, J. C. (2008). Why communication education is important: The centrality of the discipline in the 21st century. Communication Education, 57(2):224-240.

Petersen, K., Feldt, R., Mujtaba, S., and Mattsson, M. (2008). Systematic mapping studies in software engineering. In Ease, volume 8, pages 68-77.

Picard, R. W. (2000). Affective computing. MIT press.

Pretorius, R. and Budgen, D. (2008). A mapping study on empirical evidence related to the models and forms used in the uml. In Proceedings of the Second ACM-IEEE international symposium on Empirical software engineering and measurement, pages 342-344. ACM.

Scherer, K. R. (2005). What are emotions? and how can they be measured? Social science information, 44(4):695-729.

Spencer-Oatey, H. (2005). (im) politeness, face and perceptions of rapport: unpackaging their bases and interrelationships.

Terzidou, T., Tsiatsos, T., and Apostolidis, H. (2018). Architecture and interaction protocol for pedagogical-empathic agents in $3 \mathrm{~d}$ virtual learning environments. Multimedia Tools and Applications, 77(20):27661-27684.

Wang, C.-Y., Ke, S.-Y., Chuang, H.-C., Tseng, H.-Y., and Chen, G.-D. (2010). E-learning system design with humor and empathy interaction by virtual human to improve students' learning. In Proceedings of the 18th International Conference on Computers in Education. Putrajaya, Malaysia: Asia-Pacific Society for Computers in Education.(ICCE), pages 615-622.

Wang, N. and Gratch, J. (2009). Can virtual human build rapport and promote learning? In AIED, pages 737-739. 